\title{
On the Design and Performance Analysis of Low- Correlation Compact Space-Multimode Diversity Stacked Microstrip Antenna Arrays for MIMO-OFDM WLANs over Statistically-Clustered Indoor Radio Channels
}

\author{
Asuman SAVASCIHABES ${ }^{1}$, Ozgur ERTUG ${ }^{1}$, Erdem YAZGAN ${ }^{2}$ \\ ${ }^{1}$ Telecommunications and Signal Processing Laboratory, Dept. of Electrical and Electronics Engineering, \\ University of Gazi, Ankara, Turkey \\ ${ }^{2}$ Dept. of Electrical and Electronics Engineering, University of Hacettepe, Ankara, Turkey \\ ahabes@nny.edu.tr, ertug@gazi.edu.tr, yazgan@hacettepe.edu.tr
}

\begin{abstract}
The support of high spectral efficiency MIMO spatial-multiplexing communication in OFDM-based WLAN systems conforming to IEEE 802.11n standard requires the design and use of compact antennas and arrays with low correlation ports. For this purpose, compact space-multimode diversity provisioning stacked circular multimode microstrip patch antenna arrays (SCP-ULA) are proposed in this paper and their performance in terms of spatial and modal correlations, ergodic spectral efficiencies as well as compactness with respect to antenna arrays formed of vertically-oriented center-fed dipole elements (DP-ULA) and dominantmode operating circular microstrip patch antennas (CPULA) are presented. The lower spatial and modal correlations and the consequent higher spectral efficiency of SCP-ULA with ML detection over statistically-clustered Kronecker-based spatially-correlated NLOS Ricean fading channels with respect to DP-ULA and CPULA at significantly lower antenna and array sizes represents SCP-ULA as a promising solution for deployment in terminals, modems and access points of next-generation high-speed 802.11n MIMO-OFDM WLAN systems.
\end{abstract}

\section{Keywords}

IEEE 802.11n MIMO-OFDM WLAN, spectral efficiency, spatial correlation, multimode antenna, spatial-multiplexing, Kronecker channel model, NLOS Ricean fading

\section{Introduction}

In the concurrent and next-generation communication systems, the spectral efficiency and transmission quality can be vastly enhanced by multiple-input multiple-output (MIMO) communication techniques [1]. In communication systems employing MIMO spatial-multiplexing, higher data rates can be achieved when there are a large number of scatterers between the transmit and receive antennas i.e. rich-scattering environment. However, the spatial correlation between transmit and receive antenna ports that is dependent on antenna-specific parameters such as the radiation patterns, the distance between the antenna elements as well as the channel characteristics such as unfavorable spatial distribution of scatterers and angular spread severely degrades the capacity and quality achievable by MIMO spatial-multiplexing systems.

The space consumption of MIMO antennas is especially vital in applications such as access points, modems and end-user terminal equipments (laptops, PDAs etc.) of WLAN and WIMAX systems. When regularly spaced antenna elements are used in MIMO systems, the correlation between the antenna elements in a space diversity system and hence the channel capacity and transmission quality are dependent on the distance between antenna array elements, the number of antenna elements and the array geometry. However, due to the physical constraints and the concerns on ergonomics and aesthetics, the distance between antenna elements in practice cannot be extended beyond a certain level which limits the use of spaceonly diversity MIMO spatial-multiplexing systems to achieve the desired spectral efficiencies and transmission qualities. As an alternative solution to achieve compactness in MIMO systems, the use of pattern diversity [2], [3], multimode diversity [4], [5], and polarization diversity [6], [7] techniques in conjunction with space diversity are proposed in the literature.

Besides polarization diversity that is well-known, multimode and pattern diversity techniques that are less addressed in antenna engineering community are achieved by using higher-order mode generation in antenna structures and in general microstrip, biconical, helical, spiral, sinuous and log-periodic antenna structures are amenable to higher-order mode generation. In this manner, the 
higher-order modes generated in a single antenna structure with directional radiation patterns resulting in low spatial correlation in angle space are used as diversity ports in a MIMO system within a compact space. In pattern diversity on the other hand that is slightly different than multimode diversity, orthogonal radiation patterns generated on distinct antennas that are co-located at the phase-centers are generated and used as diversity ports.

In this work, a multimode stacked circular microstrip patch antenna used in a uniform linear array structure (SCP-ULA) for MIMO-OFDM WLAN systems conforming to IEEE $802.11 \mathrm{n}$ standard is designed and the associated spatial power correlation, ergodic spectral efficiency and compactness with respect to omnidirectional dipole (DP-ULA) and circular microstrip uniform linear arrays (CP-ULA) operating in the dominant isotropic TM01 mode are analyzed. Section 2 represents the 802.11n MIMOOFDM WLAN details as well as the associated system and statistically-clustered Kronecker-based correlated channel models for MIMO spatial-multiplexing. Section 3 is dedicated to the design procedure of stacked circular microstrip antenna for IEEE 802.11n MIMO-OFDM WLAN communication in HFSSv.11@TM CAD program and the analysis of the marginal and superimposition radiation patterns as well as S-parameters and VSWR variations versus frequency respectively. In Section 4, the gains of SCP-ULA with respect to CP-ULA and DP-ULA in terms of spatial/modal correlations, ergodic spectral efficiency as well as compactness are presented. Finally, Section 5 concludes the paper.

\section{IEEE 802.11N WLANS and Associated System/Channel Models}

The wireless local area network (WLAN) technology for medium-range indoor/outdoor wireless communications standardized by IEEE P802.11 working group has emerged from pre-802.11 standards towards spectrally-efficient and multipath-robust OFDM modulation based $802.11 \mathrm{~b}$ and $802.11 \mathrm{a} / \mathrm{g}$ with data rates increasing up to a maximum of $54 \mathrm{Mbps}$ for $802.11 \mathrm{a} / \mathrm{g}$. These standards are limited to the use of single transmit and receive antenna at the access points and modems as well as laptops/PDAs in WLANs for end-users forming a SISO-OFDM (single-input singleoutput OFDM) channel. In the last decade, with the proliferation of MIMO spatial-multiplexing technology using multiple transmit and receive antennas achieving much higher data rates without sacrificing either bandwidth or transmit power with respect to SISO systems, IEEE 802.11 standard is later extended with the version $802.11 \mathrm{n}$ incorporating MIMO capability with the first amendment published in 2009 [8] proposing operation at lower and upper ISM bands of $2.4 \mathrm{GHz}$ and $5.8 \mathrm{GHz}$ with the corresponding bandwidths of $20 \mathrm{MHz}$ and $40 \mathrm{MHz}$ respectively. By the use of MIMO spatial-multiplexing technology, higherorder 64/128/256-QAM modulations, a $40 \mathrm{MHz}$ channel bandwidth at $5.8 \mathrm{GHz}$ that is double that of legacy IEEE
$802.11 \mathrm{a} / \mathrm{b} / \mathrm{g}$ systems, a cyclic prefix (CP) of $400 \mathrm{~ns}$ that is half of legacy systems which reduces symbol time and hence increases data rates, more efficient OFDM structure with 52 subcarriers with respect to 48 subcarriers in legacy systems, and frame-aggregation/block-acknowledgement protocol with higher packet sizes at the MAC layer [9], [10], IEEE 802.11n standard sets forth the basis for multimedia-enabling high-throughput next-generation WI-FI networks.

The transmitter and receiver block diagrams of an IEEE $802.11 \mathrm{n}$ based MIMO-OFDM WLAN system is illustrated in Fig. 1. The binary data is first encoded by channel encoder after which the encoded bits are multiplexed into sub-streams, modulated, and transmitted from each antenna. At the receiver side, after a digital representation of $N$ received signals is obtained by $\mathrm{ADCs}$, the $\mathrm{CP}$ is removed and N-point DFT is performed per receiver branch. Since the MIMO-OFDM system turns into a narrow band flat-fading MIMO channel per sub-carrier over multipath fading channels, the received signal vector per sub-carrier is given by:

$$
\mathbf{y}(i, k)=\mathbf{H}(i) \mathbf{s}(i, k)+\mathbf{n}(i, k)
$$

where $i$ and $k$ are sub-carrier and symbol indices respectively. Here, $\mathbf{H}(i)$ represents $N \times M$ dimensional channel matrix for the $i$ th sub-carrier, $\mathbf{s}(i, k)$ represents modulated transmit symbol vectors for the $i$ th sub-carrier and $\mathbf{n}(i, k)$ represents additive white Gaussian noise at the $i$ th subcarrier and $k$ th symbol index with $N$ independent and identically distributed (i.i.d) zero-mean complex elements with variance of $\sigma_{n}^{2}$.

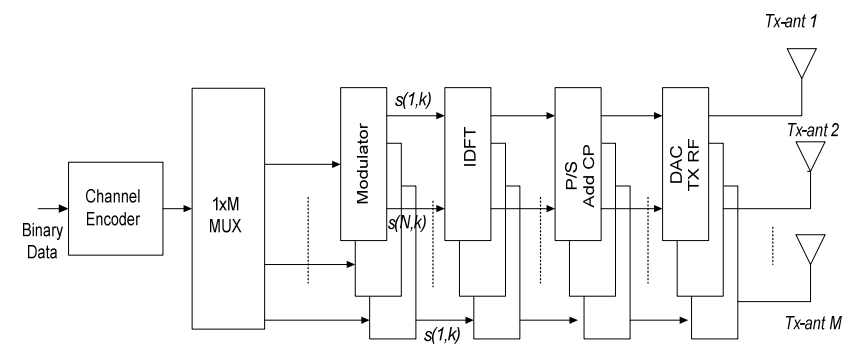

(a)

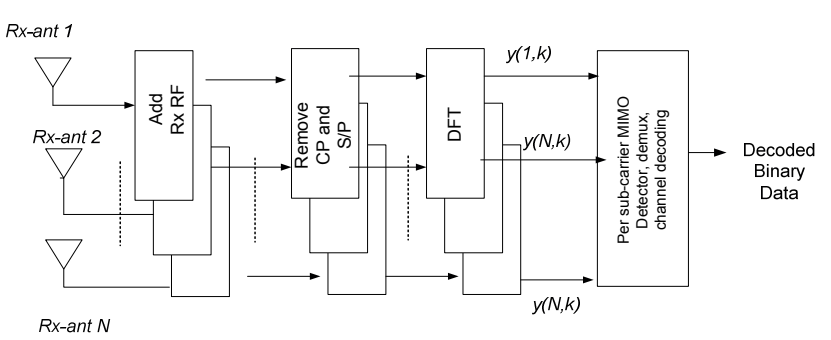

(b)

Fig. 1. Transmitter and receiver diagrams of a MIMO-OFDM WLAN system: (a) Transmitter. (b) Receiver.

Amongst the MIMO channel models proposed in the literature such as deterministic models (ray-tracing, recorded impulse response etc.) or stochastic models (geometric ring, parametric, correlation-based etc.), we deploy 
as the channel model the statistically-clustered Kronecker channel model for the characterization of the spatiallycorrelated MIMO channel in this work which has also been standardized for MIMO-OFDM IEEE 802.11n WLAN systems in [9]. Kronecker model assumes seperability between transmit/receive spatial correlations and the same model is also used for the performance analysis of indoor MIMO systems with stacked circular microstrip patch antennas with pattern diversity in [3].

The general geometry of the statistically-clustered Kronecker model we employ throughout the sequel representing the clusters and the transmission paths is represented in Fig. 2. For simplicity, only reflection from a single cluster is assumed in this work for the evaluation of the correlation and consequently spectral efficiencies, and the extension of the model to include a higher number of clusters is straightforward due to the additivity of spatial correlations.

The spatially-correlated channel matrix $\mathbf{H}_{c}$ in Kronecker model is formulated as:

$$
\mathbf{H}_{c}=\sqrt{\frac{K}{1+K}} \mathbf{H}_{L O S}+\sqrt{\frac{1}{1+K}} \mathbf{H}_{N L O S}
$$

and includes Rayleigh fading for $K=0$, Ricean fading for higher $K$ values and also AWGN channel for $K=\infty$ where $\mathbf{H}_{L O S}$ and $\mathbf{H}_{N L O S}$ are LOS (line-of-sight) and NLOS (nonlineof-sight) components respectively, $K$ is the Ricean $K$-factor given by the ratio of the LOS component's power over NLOS component's power [1] and the NLOS channel matrix is defined as [9]:

$$
\mathbf{H}_{N L O S}=\mathbf{R}_{r}^{1 / 2} \mathbf{H}_{w} \mathbf{R}_{t}^{1 / 2}
$$

where $\mathbf{H}_{w}$ denotes Wishart-type random matrix, $\mathbf{R}_{r}$ and $\mathbf{R}_{t}$ denote the transmit and receive spatial power correlation matrices respectively. The LOS component of the channel is also assumed to be rank one, and defined as [11]:

$$
\mathbf{H}_{L O S}=\mathbf{a}\left(\Omega_{\mathrm{LOS}, \mathrm{r}}\right) \cdot \mathbf{a}\left(\Omega_{\mathrm{LOS}, \mathrm{t}}\right)^{H}
$$

where $\mathbf{a}(\Omega)$ is the array response as a function of the solid angle $\Omega=(\phi, \theta)$, while $\Omega_{\mathrm{LOS}, \mathrm{t}}$ and $\Omega_{\mathrm{LOS}, \mathrm{r}}$ are AoA/AoD corresponding to the LOS component at the transmitter and receiver respectively.

\section{Design of Multimode Stacked Circular Patch (SCP) Microstrip Antenna}

The multimode stacked circular microstrip patch antenna (SCP) proposed for IEEE 802.11n MIMO-OFDM WLAN application in this work has the upper antenna in the stack excited at the TM11 mode and the bottom antenna excited at the TM21 mode to meet the compactness requirements since the radius of a circular microstrip patch antenna scales up with the mode number $m$ excited given by the formula [12]:

$$
a=\frac{\chi_{m} \lambda}{2 \pi \sqrt{\varepsilon_{r}}}
$$

where $\chi_{m}$ indicates the first zero of the derivative of the first kind Bessel function of order $m J_{m}(x)$ as presented in Tab. 1.

\begin{tabular}{|l|l|l|l|l|l|l|l|}
\hline & $\mathrm{TM}_{01}$ & $\mathrm{TM}_{11}$ & $\mathrm{TM}_{21}$ & $\mathrm{TM}_{31}$ & $\mathrm{TM}_{41}$ & $\mathrm{TM}_{51}$ & $\mathrm{TM}_{61}$ \\
\hline$\chi_{m}$ & 3.82 & 1.84 & 3.04 & 4.18 & 5.29 & 6.38 & 7.46 \\
\hline
\end{tabular}

Tab. 1. $\chi_{m}$ for different modal orders.

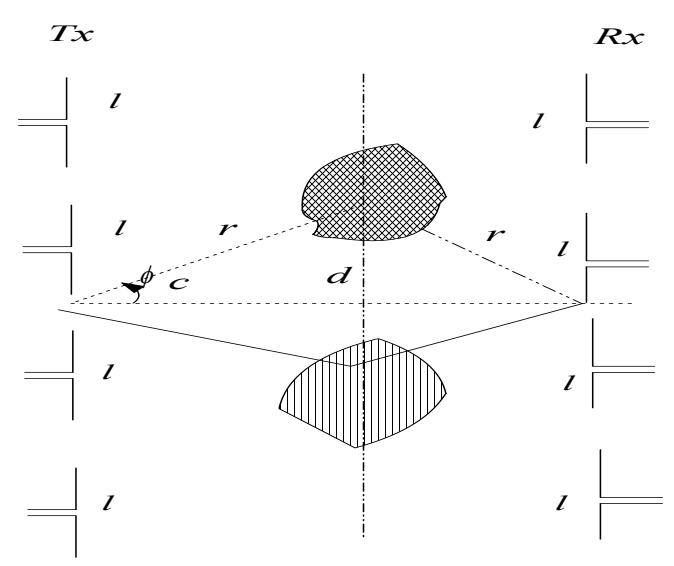

Fig. 2. Geometry of the clustered channel model representing clusters and transmit/receive antenna arrays $(4 \times 4$ array) where $\phi_{c}$ is the mean A.O.D. of the cluster.

The far-field radiation patterns for a circular microstrip patch antenna excited at the $m$-th mode is further given by [13]:

$$
\begin{aligned}
& \vec{E}_{m}=\frac{e^{-j k_{f} r}}{r}\left(E_{m, \theta} \vec{\theta}+E_{m, \phi} \vec{\phi}\right) \\
& E_{m, \theta}=\frac{j^{m} V_{m}^{0} k_{f} a}{2}\left[J_{m+1}(z)-J_{m-1}(z)\right] \cos \left(m\left(\phi-\phi_{0}\right)\right) \\
& E_{m, \phi}=-\frac{j^{m} V_{m}^{0} k_{f} a}{2}\left[J_{m+1}(z)+J_{m-1}(z)\right] \cos (\theta) \sin \left(m\left(\phi-\phi_{0}\right)\right)
\end{aligned}
$$

where $k_{f}=2 \pi / \lambda$ is the wavenumber, $J_{m}(x)$ is the Bessel function of first kind of order $m, a$ is the patch radius, $V_{m}{ }^{0}$ is the peak input voltage of the $m$ th mode, $\phi_{0}$ is the reference azimuth angle for the feed of the circular patch, and $z=k_{f} a \sin (\theta)$. Via these far-field radiation patterns, neglecting elevation spread that is acceptable for indoor propagation environments and assuming the look-direction coincident with broadside is $\theta=\pi / 2$, only $\theta$ components of the far-field radiation pattern dependent on the azimuth angle $\phi$ in (6) remains. Azimuth plane radiation patterns for mode orders $m=1,2,3$ are presented in Fig. 3 . 


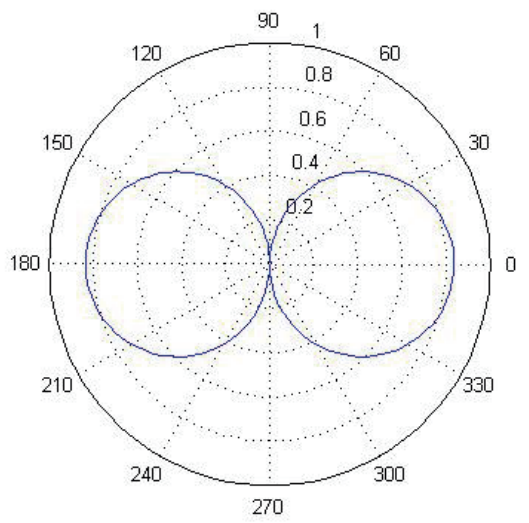

(a)

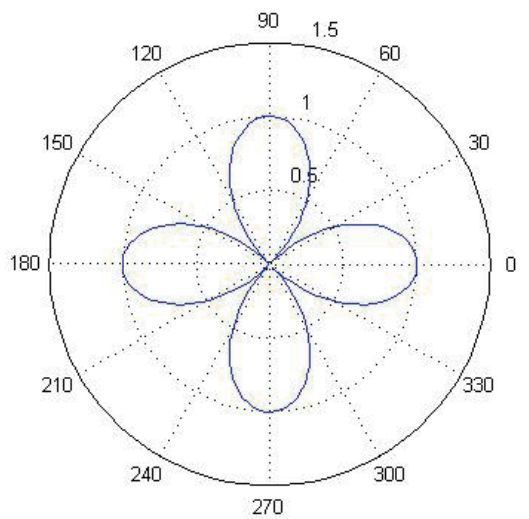

(b)

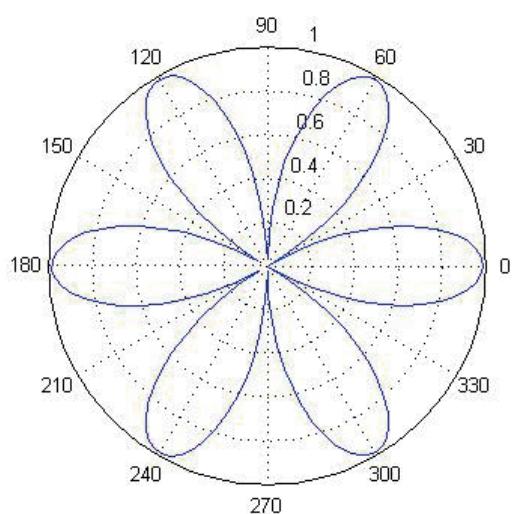

(c)

Fig. 3. Radiation patterns of multimode circular patch antennas: (a) TM11 mode, (b)TM21 mode, (c)TM31 mode.

The SCP antenna fed by microstrip feed lines designed in HFSSv.11 3D EM design and analysis software is presented in Fig. 4. To stay at the proper point of the trade-off between antenna bandwidth improvement and the antenna efficiency decrease/pattern distortion as well as to keep mutual coupling low, the distances between ground and bottom antenna as well as between circular antennas are kept as $0.5 \mathrm{~mm}$. To ensure high radiation efficiency, the dielectric constant of the substrate is further chosen low as $\varepsilon_{\mathrm{r}}=2.2$.

The theoretical radius of the top and bottom antennas in SCP antenna excited by TM11 and TM21 modes via (5)

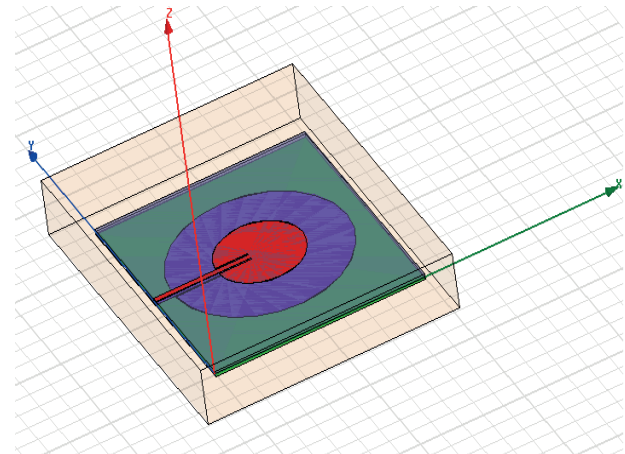

(a)

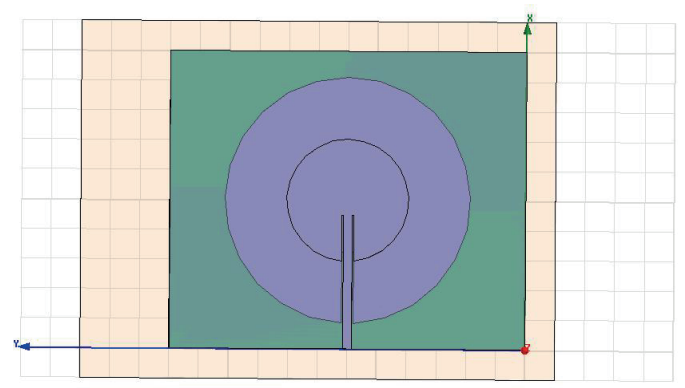

(b)

Fig. 4. The side (a) and the top (b) view of the SCP antenna with microstrip feed lines in HFSS.

are respectively given by $10.2 \mathrm{~mm}$ and $17 \mathrm{~mm}$ respectively. However, including the fringing effect [7], the actual effective radius of the circular patch antenna that is larger than estimated by (7) is obtained by the formula:

$a_{e}=a\left(1+\frac{2 h}{\pi a \varepsilon_{r}}\left(\ln \left(\frac{a}{2 h}\right)+\left(1,41 \varepsilon_{r}+1,77\right)+\frac{h}{a}\left(0,26 \varepsilon_{r}+1,65\right)\right)\right)^{1 / 2}$

through which the effective radiuses of TM11 and TM21 modes are found to be $10.6 \mathrm{~mm}$ and $17.4 \mathrm{~mm}$ respectively. To operate both stack elements at the same resonant frequency, the radius of the antenna of TM 21 mode is further modified and set as 20.67 mm in HFSSv.11@TM while the radius of the antenna operating on TM11 mode is used according to the theoretical value of $10.26 \mathrm{~mm}$.

For the impedance matching of the SCP antenna at $802.11 \mathrm{n}$ upper ISM band WLAN operating frequency of $5.8 \mathrm{GHz}$ for a $50 \Omega$ load, the radiation resistances for both stack antennas have to be evaluated first for TM11 mode and TM21 modes. The real Poynting vector is given by [13]:

$$
P+j Q=\frac{1}{2} \iint_{\text {size }}\left(\vec{E}_{a x} \vec{H}_{a}^{*}\right) \hat{z} d x d y
$$

and integrating the real part of (10):

$$
P_{r}=\frac{1}{2 \eta_{0}} \int_{0}^{2 \pi} \int_{0}^{\pi / 2}\left(\left|E_{\theta}\right|^{2}+\left|E_{\varphi}\right|^{2}\right) r^{2} \sin \theta d \theta d \varphi
$$

The relation between (11) and the radiation resistance $R_{r}$ is given by: 


$$
\begin{gathered}
P_{r}=\frac{1}{2} G_{r}\left(E_{0} h\right)^{2}=\frac{1}{2} G_{r} V_{0}^{2}, \\
R_{r}=\frac{1}{G_{r}}
\end{gathered}
$$

where $G_{r}$ is the radiation conductance. In this manner, the radiation resistance of elements operating on TM11 mode and TM21 mode are obtained as $342.57 \Omega$ and $156.13 \Omega$ respectively. In the stack antenna the radiation resistance of TM21 mode is further obtained as $131.21 \Omega$ and the radiation resistance of TM11 mode is found as $315.04 \Omega$ in HFSSv.11@TM.

Antenna input impedances are described by the equations:

$$
\begin{gathered}
Z_{\text {in }}=R_{\text {in }}+j X_{\text {in }}, \\
R_{\text {in }}=\frac{1}{G_{\text {in }}}, \\
G_{i n}=G_{r}+G_{d}+G_{c}
\end{gathered}
$$

where $Z_{i n}$, is input impedance, $R_{i n}$ is input resistance, $X_{i n}$ is input reactance, $G_{i n}$ is input conductance, $G_{d}$ is dielectric conductance and $G_{c}$ is the conductor conductance respectively. Since the antenna patch used in the design is selected as a zero-loss conductor, there exists no conductor resistance. On the other hand, the dielectric conductance value can be calculated via [14] as:

$$
G_{d}=\frac{\varepsilon_{m 0} \tan \delta}{4 \mu_{0} h f_{r}}\left[\left(k a_{r}\right)^{2}-m^{2}\right]
$$

where $\varepsilon_{m 0}=2$ for $m=0$ and $\varepsilon_{m 0}=1$ for $m \neq 0$, and $h$ is height of dielectric substrate of microstrip line. The effective loss tangent of the dielectric material with $\varepsilon_{r}=2.2$ is determined as $\delta=0.0009$. Using these values, the dielectric conductances for TM11 and TM21 modes are obtained as $G_{d}=1.4925 \times 10^{-4}$ and $G_{d}=6.0936 \times 10^{-4}$ respectively. These values are quite small so that it is rational not to take dielectric conductances into account for $G_{\text {in }}$ calculations and to state that the input resistances of the antenna operating at TM1 1 mode is $342.57 \Omega$, and the input resistance of the antenna operating at TM21 mode is $156.13 \Omega$. To adapt these resistance values to $50 \Omega$, microstrip feed line widths for both elements in the stacked antenna are determined as $1.5 \mathrm{~mm}$ via the guideline equations in [15] such that both stack elements are matched to $50 \Omega$ input resistance.

In the design of SCP antenna, the antenna reactances are further calculated via

$$
\begin{aligned}
& X_{\text {in }}=-j w \mu_{0} h\left[\frac{1}{\pi a_{e}^{2} k^{2}}+\right. \\
& \left.\sum_{m=2}^{\infty} \frac{j_{0}^{2} k_{0 m} \rho_{0}}{\pi a^{2} j_{0}^{2} k_{0 m} a_{e}\left(k^{2}-k_{0 m}^{2}\right)}+\frac{2}{\pi} \sum_{m=1}^{\infty}\left(\frac{\sin (n \Delta)}{n \Delta}\right)^{2}\right]
\end{aligned}
$$

and found as $-\mathrm{j} 162.7 \Omega$ and $-\mathrm{j} 107.13 \Omega$ for TM11 and TM21 modes respectively. In the stack antenna, the evaluation of the reactances of antenna with TM21 mode is obtained as $\mathrm{j} 77.316 \Omega$ while that of TM11 mode is found as $j 123.25 \Omega$ that are quite high values for matching and can be minimized by optimizing the length of the microstrip feed lines.

The characteristic impedance of the feed line can be found depending on the size of the microstrip feed line by using:

$$
Z_{0}=\left\{\begin{array}{cc}
\frac{\eta}{2 \pi \sqrt{\varepsilon_{r e}}} \ln \left(\frac{8 h}{L}+0.25 \frac{L}{h}\right), & (L / h \leq 1) \\
\frac{\eta}{\sqrt{\varepsilon_{r e}}}\left\{\frac{L}{h}+1.393+0.667 \ln \left(\frac{L}{h}+1.444\right)\right\}^{-1} & (L / h \geq 1)
\end{array}\right.
$$

where $L$ is microstrip feed line width. Effective dielectric constant is also calculated via:

$$
\varepsilon_{r e}=\frac{\varepsilon_{r e}+1}{2}+\frac{\varepsilon_{r e}-1}{2} F(L / h) .
$$

According to the desired characteristic impedance value, the ratio of the microstrip feedline width $L$ to dielectric substrate height $h$ is calculated via:

$$
\frac{L}{h}=\left\{\begin{array}{cc}
\frac{8 \exp (A)}{\exp (2 A)-2}, & (L / h<2) \\
\left\{B-1-\ln (2 B-1)+\frac{\varepsilon_{r}-1}{2 \varepsilon_{r}}\left[\ln (B-1)+0.39-\frac{0.61}{\varepsilon_{r}}\right]\right\}, & (L / h \geq 1)
\end{array}\right.
$$

where $A$ and $B$ are:

$$
\begin{aligned}
& A=\frac{Z_{0}}{60} \sqrt{\frac{\varepsilon_{r}+1}{2}}+\frac{\varepsilon_{r}-1}{\varepsilon_{r}+1}\left(0.23+\frac{0.11}{\varepsilon_{r}}\right), \\
& B=\frac{377 \pi}{2 Z_{0} \sqrt{\varepsilon_{r}}} .
\end{aligned}
$$

With the height of the dielectric substrate $h=0.5 \mathrm{~mm}$, the line width $L$ is calculated as $1.55 \mathrm{~mm}$ via (22) for the microstrip feed lines and in this manner, the antenna reactances of TM11 mode and TM21 modes are minimized to $\mathrm{j} 11.513 \Omega$ and $\mathrm{j} 4.5072 \Omega$ respectively with the corresponding antenna input impedances finally obtained and used in S-parameter and VSWR analysis as $Z_{\text {in } 1}=45.507+\mathrm{j} 11.513 \Omega$ and $Z_{\text {in } 2}=50.678+\mathrm{j} 4.5072 \Omega$ for TM11 and TM21 modes respectively.

The S-parameters versus frequency of the designed SCP antenna are analyzed in Fig. 5. The resonance of both modes are achieved around $5.8 \mathrm{GHz}$ that is suitable for IEEE 802.11n MIMO-OFDM WLANs operating in upperISM band and the operating bandwidth of SCP antenna is measured to be around $55 \mathrm{MHz}$ via $\mathrm{S} 11$ and S22 returnloss S-parameters that is sufficiently adequate for IEEE 802.11n WLAN communication which requires $40 \mathrm{MHz}$ 


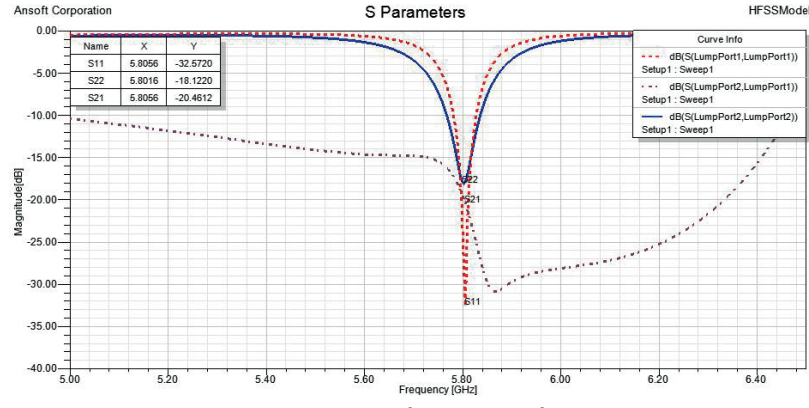

Fig. 5. S-parameters versus frequency of SCP antenna.

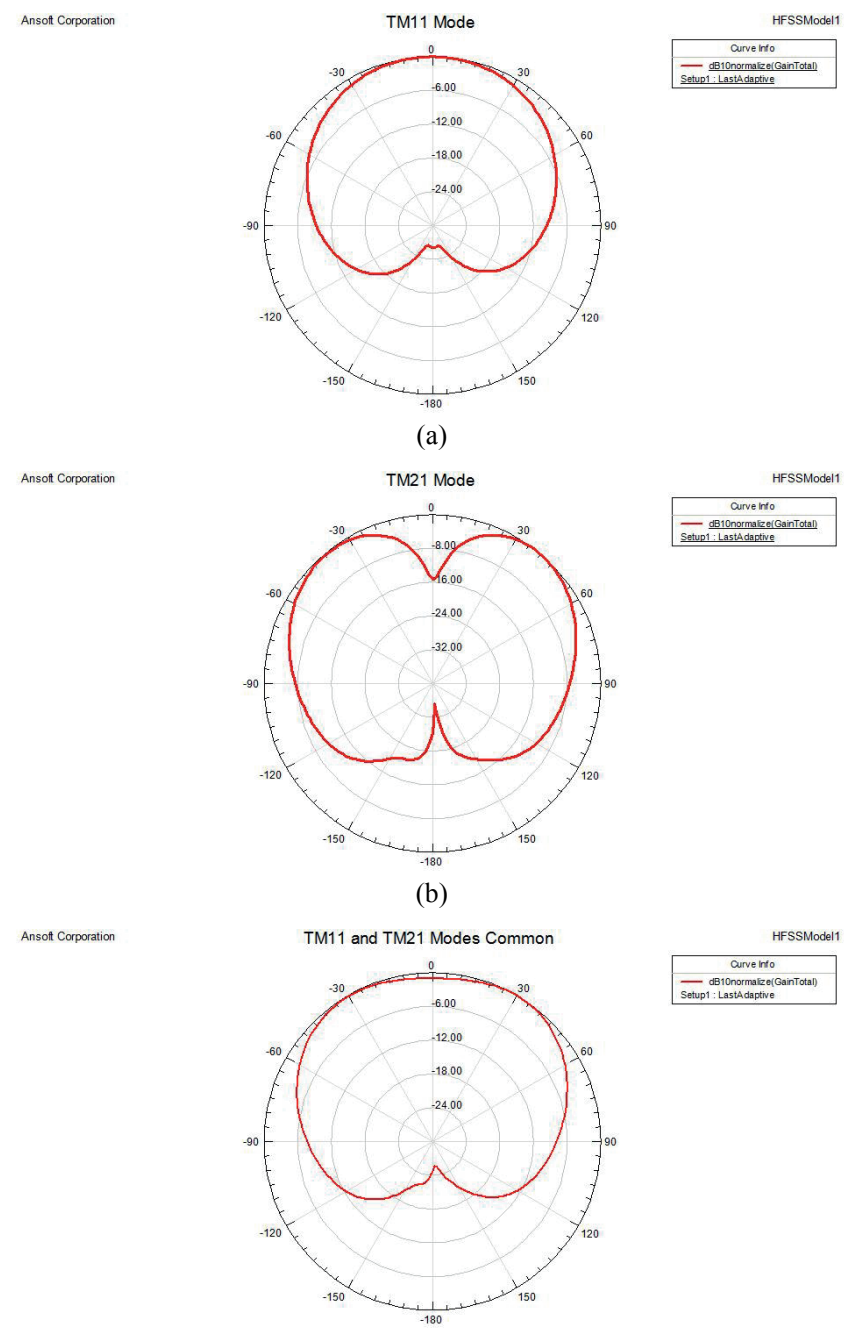

(c)

Fig. 6. Radiation patterns of SCP antenna: (a) TM11 alone, (b) TM21 alone, (c) TM11 andTM21 superimposed.

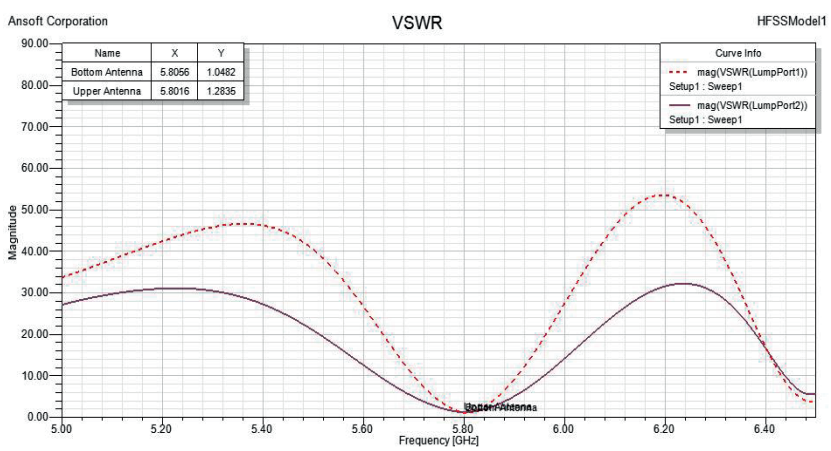

Fig. 7. VSWR plots versus frequency for SCP antenna. bandwidth. Furthermore, the S21 mutual-coupling S-parameter in Fig. 4 is well below approximately $-20 \mathrm{~dB}$ within the operating bandwidth around $5.8 \mathrm{GHz}$ letting the omittance of pattern distortion due to the coupling effects between the collocated antennas. This fact is also well apparent in Fig. 6 where the radiation patterns of both modes are presented marginally and in superimposed form. Due to the low mutual coupling achieved, the radiation pattern distortion imposed by TM21 mode on TM11 mode is negligible in the superimposed radiation pattern in Fig. 6(c).

The VSWR plots of the SCP antenna for both TM11 and TM21 modes versus frequency are further presented in Fig. 7. The VSWR values for the TM11 and TM 21 modes at the resonance frequency of $5.8 \mathrm{GHz}$ is measured in HFSSv.11@TM as 1.0482 and 1.2835 respectively that are very close to the theoretical lower bound of unity validating the reliability of the impedance matching of SCP antenna.

\section{Spatial/Modal Correlations and Spectral Efficiency Analysis of SCP-ULA}

To evaluate the achievable gains with multimode SCP antenna used as a uniform linear array (SCP-ULA) for IEEE $802.11 \mathrm{n}$ MIMO-OFDM WLAN communications when employed at access points, modems or end-user terminal equipments, the spatial/modal power correlations of the SCP-ULA is compared with the spatial/modal power correlations of uniform linear arrays of center-fed dipole antennas of length $\lambda / 2$ (DP-ULA) and the spatial/modal power correlations of uniform linear arrays of isotropic TM01 dominant-mode operating circular patch antennas in Fig. 8 for a statistically-clustered indoor propagation environment with a single-cluster of angular spread $\sigma_{c}^{2}$ and uniformly-distributed mean angle of arrival (AoA) and angle of departure $(\mathrm{AoD})$ with respect to array broadsides. The spatial/modal normalized complex correlation coefficient of all three types of antennas is given by:

$\rho=\frac{\int_{-\pi}^{\pi} E_{1}(\phi) E_{2}^{*}(\phi) P A S\left(\phi ; \phi_{c}, \sigma_{c}^{2}\right) \exp \left(-j k_{f} d\left(n_{2}-n_{1}\right) \sin (\phi)\right) d \phi}{\sqrt{\int_{-\pi}^{\pi}\left|E_{1}(\phi)\right|^{2} P A S\left(\phi ; \phi_{c}, \sigma_{c}^{2}\right) d \phi} \cdot \sqrt{\int_{-\pi}^{\pi}\left|E_{2}(\phi)\right|^{2} P A S\left(\phi ; \phi_{c}, \sigma_{c}^{2}\right) d \phi}}$

and the power azimuth spectrum $P A S\left(\phi ; \phi_{c}, \sigma_{c}^{2}\right)$ that defines the distribution of power over the sub-multipath components within a cluster is used as truncated-Laplacian density with mean azimuth angle $\phi_{c}$ and angular spread $\sigma_{c}^{2}$ the form of which is given by [16]:

$$
P A S\left(\phi ; \phi_{c}, \sigma_{c}^{2}\right)=\frac{e^{-\left|\frac{\sqrt{2}\left(\phi-\phi_{c}\right)}{\sigma_{c}}\right|}}{\sqrt{2} \sigma_{c}\left(1-e^{-\frac{\sqrt{2} \pi}{\sigma_{c}}}\right)} .
$$




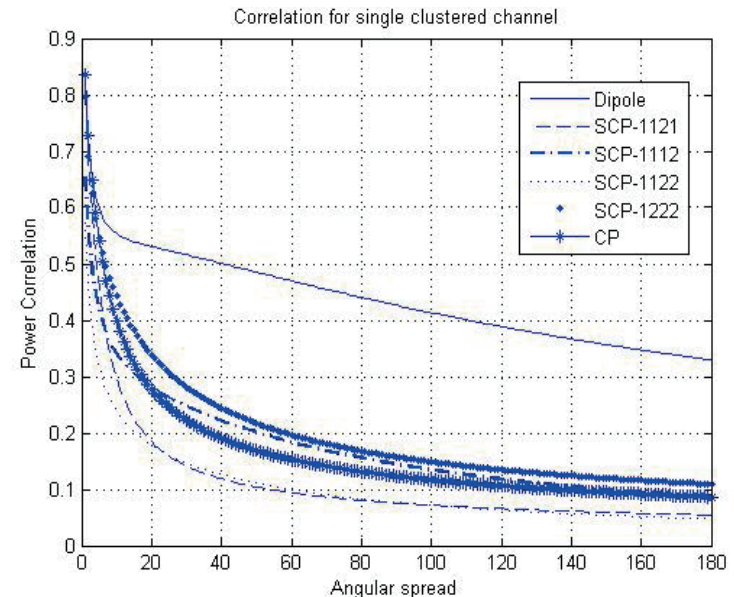

Fig. 8. Power correlation values of DP-ULA, CP-ULA and SCP-ULA averaged over azimuth angle $\phi$ for a $2 \times 2$ array.

The power correlation value that is strictly related to SNR scaling for MIMO channels is then given by $\rho_{p}=|\rho|^{2}$ and the power correlation values are averaged over uniformly-distributed mean azimuth angles over azimuth angle $\phi$.

The power correlation values of the SCP antenna for all angular spread values and spatial/modal combinations in the densest configuration where stack antennas are nearby in the most compact scenario allowed by the physical radius of the bottom antenna for $2 \times 2$ configuration as presented in Fig. 8 are much lower than that of the two nearest antennas spaced at the patch radius of SCP bottom antenna in $\lambda / 2$ - length DP-ULA and CP-ULA which dictates spectral efficiency gains for MIMO spatial-multiplexing.

In Fig. 9, the power correlations of SCP-ULA versus mean azimuth angle for two different values of low $\left(5^{\circ}\right)$ and high $\left(20^{\circ}\right)$ angular spread are presented. For all angular spread values, DP-ULA has the maximum correlation values in comparison with $\mathrm{CP}$ and SCP at broadside, where the cluster is at the center of the antenna arrays and the oscillations of the patch antennas SCP-1122 (upper antenna radiated at TM11 and lower antenna radiated at TM22 mode), and SCP-1222 (upper antenna radiated at TM12 and lower antenna radiated at TM22 mode) decrease directly with the increase in angular spread. Besides, the maximum value of the correlations for all antennas is inversely proportional with angular spread.

The ergodic spectral efficiencies in bps/Hz of MIMOOFDM WLAN system with DP-ULA, CP-ULA and SCPULA versus angular spread for $2 \times 2,4 \times 4,6 \times 6$ and $8 \times 8$ configurations are further presented in Fig. 10 over singlecluster Kronecker-model NLOS Rayleigh fading channel via [17]:

$$
\eta_{e}=E\left\{\frac{1}{N_{c}} \sum_{i=1}^{N_{c}} \log _{2}\left(\operatorname{det}\left(\mathbf{I}_{N}+\frac{S N R}{M} \mathrm{H}_{c} \mathrm{H}_{c}{ }^{H}\right)\right)\right\}
$$

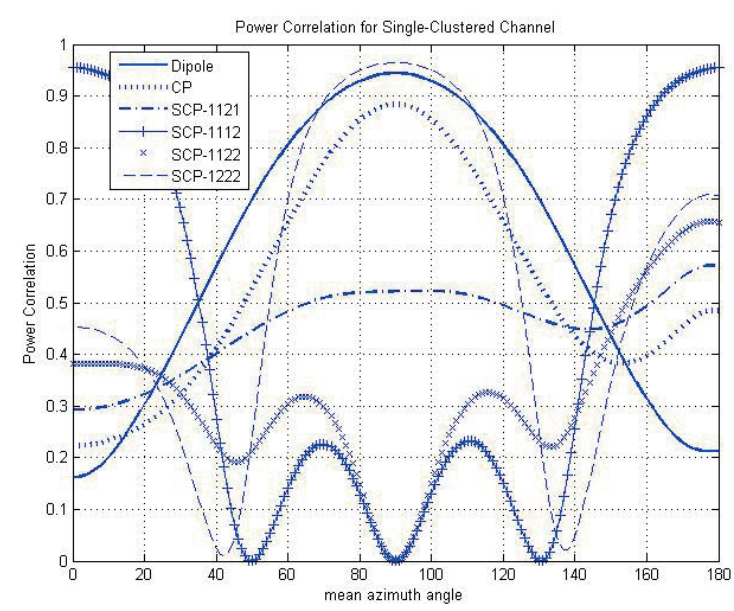

(a)

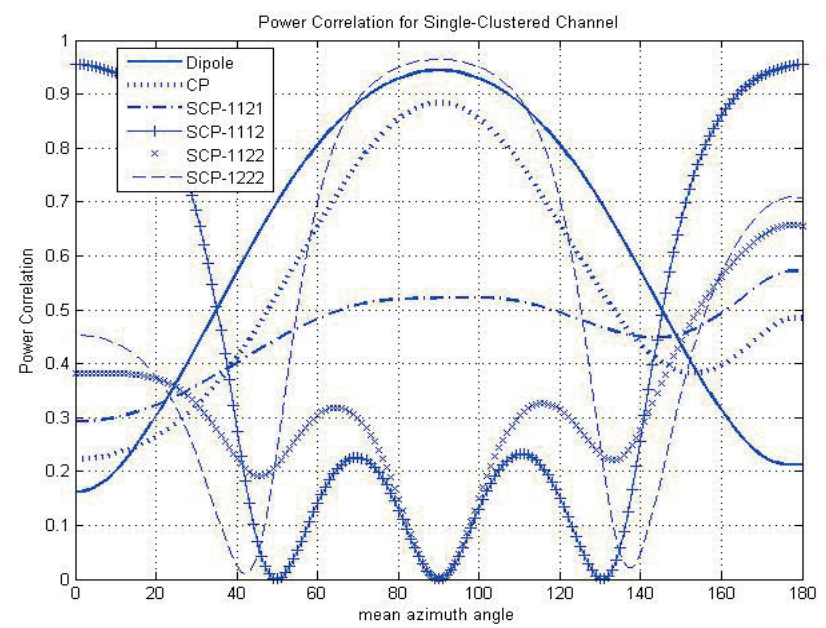

(b)

Fig. 9. Power correlation values of DP-ULA, CP-ULA and SCP-ULA versus mean azimuth angle (a): $\sigma_{c}^{2}=5^{\circ}$, (b) $\sigma_{c}^{2}=20^{\circ}$

where the correlated channel matrix $\mathbf{H}_{c}$ has the Kronecker form [18-20]; $\mathbf{H}_{c}=\mathbf{R}_{R X}^{1 / 2} \mathbf{H}_{w} \mathbf{R}_{T X}^{1 / 2}$, in terms of the receive and transmit normalized power correlation matrices $\mathbf{R}_{R X}$ and $\mathbf{R}_{T X}$ and the elementwise-independent $N \times M$ Wisharttype random matrix $\mathbf{H}_{w}$ with elements distributed as CN(0.1).

For $2 \times 2$ and $4 \times 4$ configurations, the SCP-ULA has much higher spectral efficiency than DP-ULA and slightly higher spectral efficiency than CP-ULA. On the other hand, for $6 \times 6$ and $8 \times 8$ configurations, CP-ULA and SCPULA have nearly the same spectral efficiencies which is also higher than DP-ULA. Furthermore, based on the spectral efficiency results presented in Fig. 10, data rates achievable with SCP-ULA conforming to IEEE $802.11 \mathrm{n}$ standards with $40 \mathrm{MHz}$ bandwidth at $5.8 \mathrm{GHz}$ for $2 \times 2$, $4 \times 4,6 \times 6$ and $8 \times 8$ array configurations are $164 \mathrm{Mbps}$, $324 \mathrm{Mbps}$, $480 \mathrm{Mbps}$, $644 \mathrm{Mbps}$ respectively for high angular spreads that are much higher than standard $54 \mathrm{Mbps}$ data rate achievable with SISO-OFDM $802.11 \mathrm{a} / \mathrm{g}$ WLAN systems. 
The ergodic spectral efficiency of SCP-ULA, CP-ULA and DP-ULA versus mean azimuth angle of the cluster with respect to the antenna array boresights are further presented in Fig. 11 for low and high angular spreads of $\sigma_{c}^{2}=50$ and $\sigma_{c}^{2}=200$ respectively. In both cases, the ergodic spectral efficiency of SCP-ULA is the highest when the cluster is at the boresight; i.e. $\phi_{c}=90^{\circ}$ followed by CP-ULA and DP-ULA. On the other hand, as the cluster moves towards endfires with respect to the

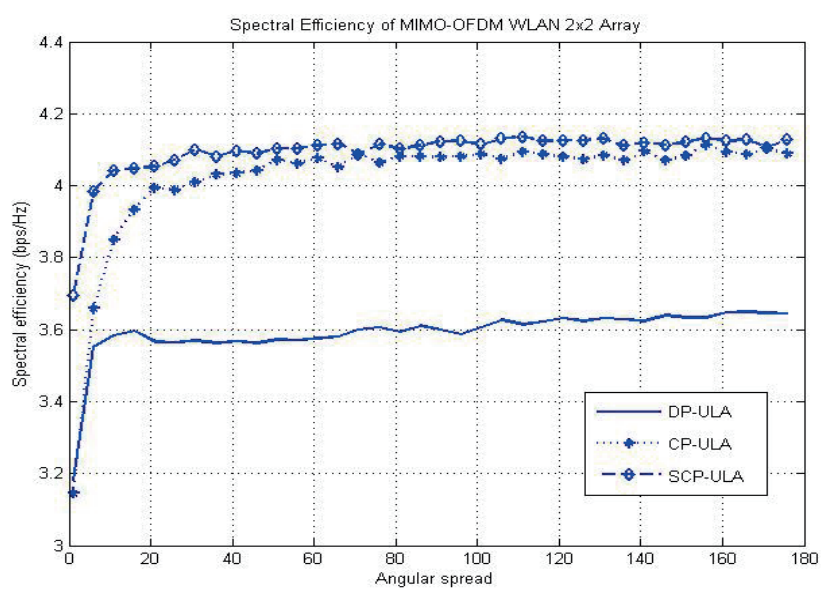

(a)

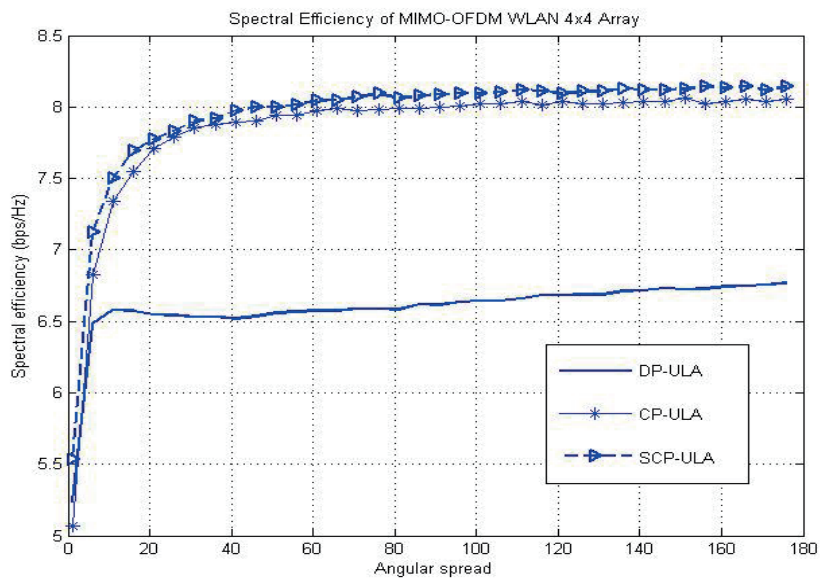

(b)

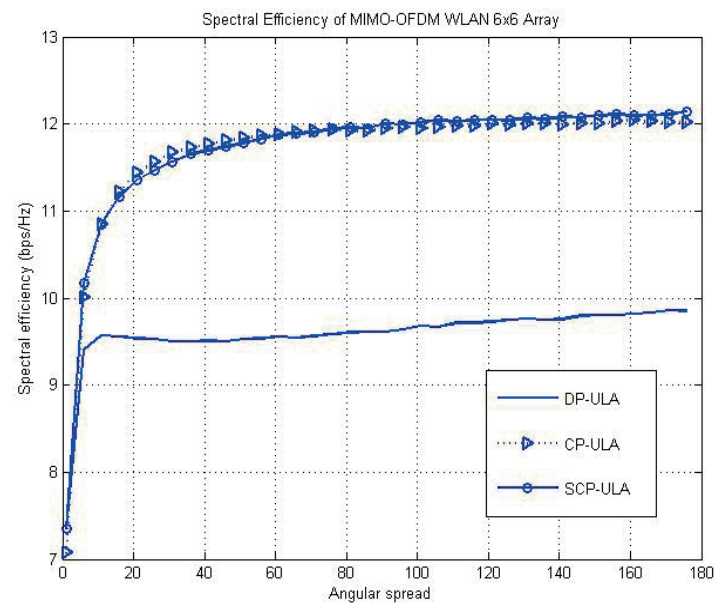

(c)

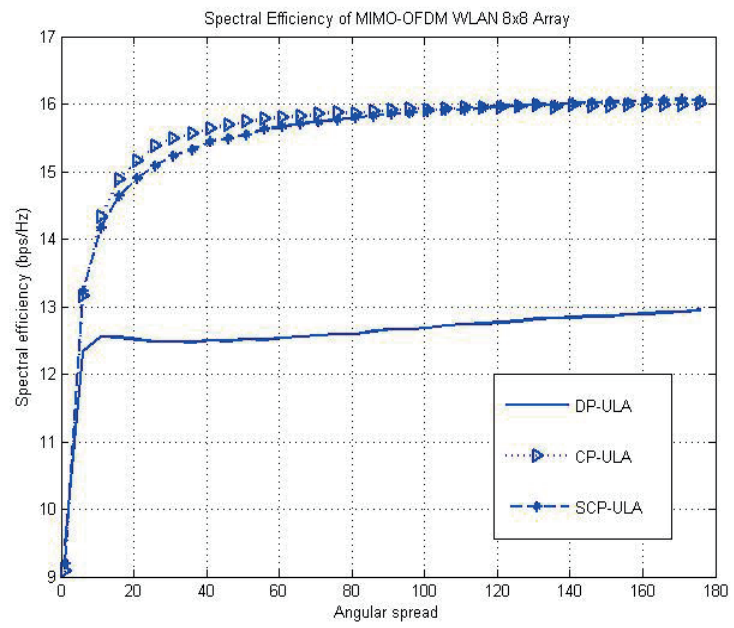

(d)

Fig. 10. Ergodic spectral efficiency of MIMO-OFDM WLAN over Kronecker-based statistically-clustered singlecluster NLOS Rayleigh fading channel with DP-ULA, CP-ULA and SCP-ULA versus angular spread at $\mathrm{SNR}=10 \mathrm{~dB}$ : (a) $2 \times 2$, (b) $4 \times 4$, (c) $6 \times 6$, (d) $8 \times 8$ array.

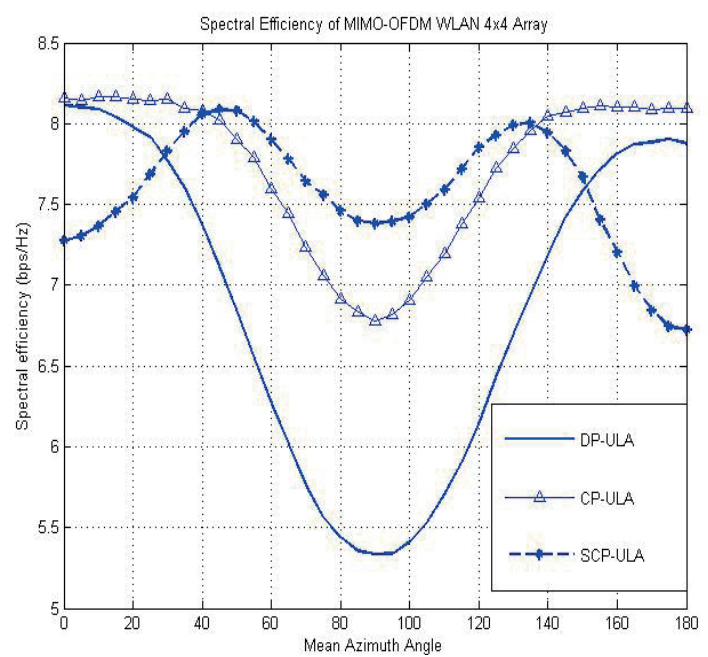

(a)

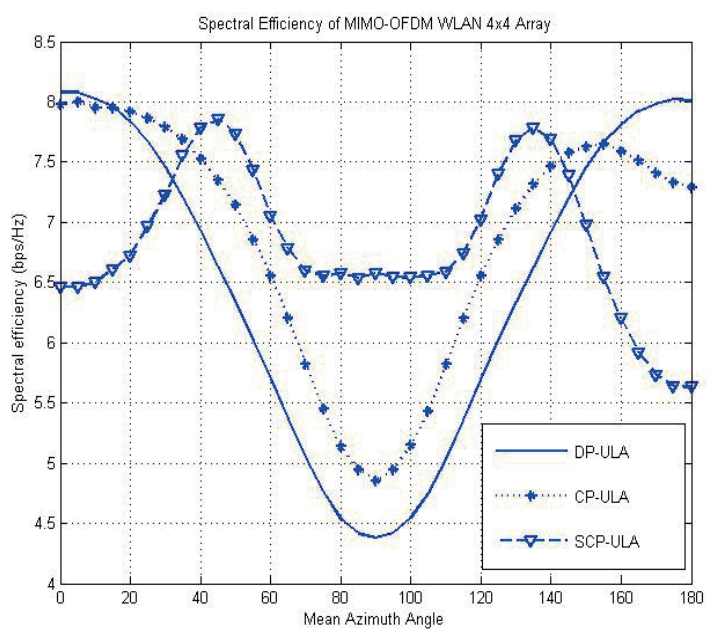

(b)

Fig. 11. Ergodic spectral efficiency of MIMO-OFDM WLAN over NLOS fading channel versus mean azimuth angle for $4 \times 4$ configuration of DP-ULA, CP-ULA and SCPULA at (a) $\sigma_{c}^{2}=5^{\circ}$, (b) $\sigma_{c}^{2}=20^{\circ}$. 
antenna array boresights; i.e. $\phi_{c}=0^{\circ}$ and $\phi_{c}=180^{\circ}, \mathrm{CP}-$ ULA and DP-ULA respectively presents higher spectral efficiencies with respect to SCP-ULA. Despite this lower spectral efficiency of SCP-ULA with respect to CP-ULA and DP-ULA at endfires, the clusters in practical scenarios are mainly concentrated around boresight of antenna arrays in indoor propagation mediums and the range of mean azimuth angles around boresight over which SCP-ULA dominates CP-ULA and DP-ULA in terms of spectral efficiency is where the clusters reside realistically.

Most importantly, these lower correlation and higher spectral efficiency gains are achieved at $33.3 \%$ and $49.9 \%$, $40 \%$ and $50 \%$, and $42,9 \%$ and $50 \%$ denser space in compactness with SCP-ULA with respect to DP-ULA and CPULA for $4 \times 4,6 \times 6$ and $8 \times 8$ configurations respectively as tabulated in Tab. 2 and Tab. 3.

\begin{tabular}{|l|c|c|c|c|}
\hline$($ Type $) /(\mathrm{N} \times \mathrm{M})$ & $2 \times 2(\%)$ & $4 \times 4(\%)$ & $6 \times 6(\%)$ & $8 \times 8(\%)$ \\
\hline DP-ULA & 0 & 33.3 & 40.0 & 42.9 \\
\hline CP-ULA & 49.7 & 49.9 & 50.0 & 50.0 \\
\hline
\end{tabular}

Tab. 2. Compactness gain of SCP-ULA with respect to CP-ULA and DP-ULA.

\begin{tabular}{|c|c|c|c|c|}
\hline$(\mathrm{TYPE}) /(\mathrm{N} \times \mathrm{M})$ & $\begin{array}{c}2 \times 2 \\
(\mathrm{~mm})\end{array}$ & $\begin{array}{c}4 \times 4 \\
(\mathrm{~mm})\end{array}$ & $\begin{array}{c}6 \times 6 \\
(\mathrm{~mm})\end{array}$ & $\begin{array}{c}8 \times 8 \\
(\mathrm{~mm})\end{array}$ \\
\hline DP-ULA & 21.2 & 63.6 & 106 & 148.4 \\
\hline CP-ULA & 42.4 & 84.8 & 127.2 & 169.6 \\
\hline SCP-ULA & 16.9 & 33.8 & 50.7 & 67.6 \\
\hline
\end{tabular}

Tab. 3. Physical array length of SCP-ULA, CP-ULA and DPULA for minimum distance between elements.

\section{Conclusions}

In this paper, we proposed space-multimode stacked circular patch uniform linear antenna arrays (SCP-ULA) for deployment in size-constrained access points, modems and end-user terminal equipments of IEEE $802.11 \mathrm{n}$ MIMO-OFDM WLAN systems at $5.8 \mathrm{GHz}$. The performance of SCP-ULA in terms of spatio-modal power correlations, ergodic spectral efficiencies and compactness are compared with these of dominant-mode operating circular patch antenna arrays (CP-ULA) and center-fed dipole arrays (DP-ULA). The higher or comparable spectral efficiencies and lower spatio-modal correlations achieved by SCP-ULA over Kronecker-based statistically-clustered single-cluster NLOS Rayleigh fading channels in much more compact space requirements present space-multimode SCP-ULA antennas as a favorable solution for deployment in spatially-compact equipments of next-generation highspeed indoor IEEE 802.11n MIMO-OFDM WLAN systems.

\section{References}

[1] TELATAR, I. E. Capacity of multi-antenna Gaussian channels. European Transactions on Telecommunications, 1999, vol. 10, no. 6 , p. 585-596. DOI: $10.1002 /$ ett.4460100604
[2] FOSCHINI, G. J., GANS, M. On limits of wireless communications in a fading environment when using multiple antennas. Wireless Personal Communications, 1998, vol. 6, p. 311-355. DOI: $10.1023 / \mathrm{A}: 1008889222784$

[3] FORENZA, A., HEATH, R. W. Benefit of pattern diversity via two-element array of circular patch antennas in indoor clustered MIMO channels. IEEE Transactions on Communications, 2006, vol. 54, no. 5, p. 943-954. DOI: 10.1109/TCOMM.2006.873978

[4] SANCHEZ-FERNANDEZ, M., RAJO-IGLESIAS, E., QUEVEDO-TERUEL, O., PABLO-GONZALEZ, M. L. Spectral efficiency in MIMO systems using space and pattern diversities under compactness constraints. IEEE Transactions on Vehicular Technology, 2008, vol. 57, no. 3, p. 1637-1645. DOI: 10.1109/TVT.2007.909279

[5] SVANTESSON, T. Correlation and channel capacity of MIMO systems employing multimode antennas. IEEE Transactions on Vehicular Technology, 2002, vol. 51, no. 6, p. 1304-1312. DOI: 10.1109/TVT.2002.804856

[6] MUKHERJEE, A., KWON, H. M. Compact multi-user wideband MIMO system using multiple-mode microstrip antennas. In Proceedings of $65^{\text {th }}$ Vehicular Technology Conference VTC 2007 Spring. Dublin (Ireland), April 2007, p. 584-588. DOI: 10.1109/VETECS.2007.131

[7] WALDSCHMIDT, C., KUHNERT, C., SCHULTEIS, S. WIESBECK, W. Compact MIMO-arrays based on polarizationdiversity. In Proceedings of IEEE Antennas and Propagation Symposium 2003. Columbus (USA), 2003, vol. 2, p. 499-502. DOI: $10.1109 /$ APS.2003.1219284

[8] IEEE 802.11n-2009-Amendment 5: Enhancements for Higher Throughput. IEEE-SA. 29 October 2009. DOI: 10.1109/IEEESTD.2009.5307322.

[9] IEEE P802.11n Wireless LANs: TGn Channel Models. IEEE-SA. May 10 2004. doc.: IEEE 802.11-03/940r4.

[10] Wi-Fi CERTIFIED n: Longer-Range, Faster-Throughput, Multimedia-Grade Wi-Fi® Networks, Wi-Fi Alliance. Sept. 2009.

[11] PAULRAJ, A., NABAR, R., GORE, D. Introduction to SpaceTime Wireless Communications. New York: Cambridge University Press, 2003.

[12] SVANTESSON, T. On the capacity and correlation of multiantenna systems employing multiple polarizations. In Proc. of IEEE Antennas and Propagation Symposium 2002. San Antonio (USA), 2002, vol. 3, p. 202-205. DOI: 10.1109/APS. 2002.1018190

[13] VAUGHAN, R. G. Two-port higher mode circular microstrip antennas. IEEE Transactions on Antennas and Propagation, 1988, vol. 36, no. 3, p. 309-321. DOI: 10.1109/8.192112

[14] GARG, R., BHARTIA, P., BAHL, I. Microstrip Antenna Design Handbook. Artech House, 2001.

[15] BALANIS, C.A. Antenna Theory: Analysis and Design. $2^{\text {nd }}$ ed. United States of America: John Wiley \& Sons Inc., 1997.

[16] BAHL, J. Lumped Elements for RF and Microwave Circuits. Boston: Artech House, 2003.

[17] VAN ZELST, A., VAN NEE, R., AWATER, G. A. Space-division multiplexing for OFDM systems. In IEEE Vehicular Technology Conference 2000. Tokyo (Japan), 2000, vol. 2, p. 1070-1074. DOI: 10.1109/VETECS.2000.851289

[18] CHE-NEE CHUAH, KAHN, J. M., TSE, D. N. C. Capacity of multi-antenna array systems in indoor wireless environments. In Proceedings of GLOBECOM'98. Sidney (Australia), 1998, vol. 4, p. 1894-1899. DOI: 10.1109/GLOCOM.1998.775873

[19] GOLDSMITH, A. MIMO Wireless Communications. Cambridge University Press, 2007. 
[20] VAN ZELST, A. Space-division multiplexing algorithms. In 10th Mediterranean Electrotechnical Conference 2000, MELECON 2000. Cyprus, 2000, vol. 3, p. 1218-1221. DOI: 10.1109/MELCON.2000.879755

\footnotetext{
About the Authors ...

Asuman SAVAŞCIHABEŞ has received her Ph.D. degree in Electrical Engineering in 2013 specializing on Telecommunications and Signal Processing at Gazi University, Ankara, Turkey. She is currently working as an assistant professor in Electrical and Electronics Engineering Department of Nuh Naci Yazgan University, Kayseri, Turkey. Her research interests include wireless communication systems, MIMO communication and transmit/receive diversity of MIMO-OFDM.

Özgür ERTUG was born in Ankara, Turkey in 1975. He received his B.Sc. degree in 1997 from University of Southern California, USA, M.Sc. degree from Rice University in 1999 and Ph.D. degree from Middle East Tech-
}

nical University in 2005 . He is currently working as an assistant professor in Electrical and Electronics Engineering Department of Gazi University. His main research interests lie in algorithm and architecture design as well as theoretical and simulation-based performance analysis of wireless communication systems especially in the physical layer.

Erdem YAZGAN received the B.S. and M.S. degrees from the Middle East Technical University, Ankara, Turkey, in 1971 and 1973, respectively, and the Ph.D. degree from Hacettepe University, Ankara, Turkey, in 1980, all in Electrical Engineering. Since 1990, she has been a Professor with the Department of Electrical Engineering, Hacettepe University. In 1989, she was a Visiting Professor with Essex University, Essex, U.K. In 1994, she was with the Electroscience Laboratory, Ohio State University, Columbus. Her research interests include HF propagation, low altitude radar systems, mobile communications, MICs, reflector and microstrip antennas, Gaussian beam solutions, conformal mapping, and medical electronics. 